\title{
A renal genetics clinic - model for our times
}

\author{
Authors: Caroline M Robinson, ${ }^{\mathrm{A}}$ Richard N Sandford, ${ }^{\mathrm{A}, \mathrm{B}}$ Oliver Wiseman, ${ }^{\mathrm{C}}$ Anita Sarker ${ }^{\mathrm{D}}$ and Fiona E Karet Frank $\mathrm{B}^{\mathrm{B}, \mathrm{E}}$
}

\section{Aims}

Significant advances in the technology, cost and speed of processing, and interpretation of complex genetic information are offering new opportunities to incorporate genetics and genomics into routine healthcare; here we evaluated our multidisciplinary renal genetics clinic to provide insights into how similar services, incorporating genetics expertise embedded within other medical specialties, might be established.

\section{Methods}

This was the first specialist renal genetics clinic in the UK; we considered the important elements of the service offered to patients and families with known, or suspected, inherited renal conditions. We used patient records to examine demographics and satisfaction surveys to assess impact.

\section{Results}

From inauguration in 2005 to the end of 2017, the weekly clinic has managed nearly 1,900 patients with inherited or heritable renal disorders, individually or as part of an affected family (Table 1). Specialties involved are nephrology, clinical genetics, clinical biochemistry and urology, with specialist nursing support. We found that some patients with rare disorders are prepared to travel significant distances to attend.

Two patient surveys (2006/2011) were very positive, demonstrating high levels of satisfaction with both staff and the service provided: $97 \%(n=170)$ and $97.7 \%(n=145)$ respectively rated the service overall as 'excellent' or 'good', confirming that quality has been maintained over time.

Patients particularly appreciated:

$>$ the quality of tailored information and specialist expertise in inherited renal disorders, ranging from polycystic kidney disease to ultra-rare (eg Dent disease)

$>$ the range and continuity of care provided within one clinic, from pre-screening genetic counselling through investigative testing (including relevant genetic testing), to long-term regular clinical monitoring until the patient requires renal replacement therapy

Authors: ${ }^{A}$ Clinical Genetics, Cambridge University Hospitals NHS Foundation Trust, Cambridge, UK; ${ }^{\mathrm{B}}$ Medical Genetics, University of Cambridge, Cambridge, UK; ${ }^{C}$ Urology; ${ }^{\mathrm{D}}$ Clinical Biochemistry; ${ }^{E}$ Nephrology, Cambridge University Hospitals NHS Foundation Trust, Cambridge, UK

\section{Table 1. Range of conditions managed in the} multidisciplinary renal clinic

\section{Condition}

Nephrolithiasis /

nephrocalcinosis /

medullary sponge

kidney

Autosomal dominant polycystic kidney

disease

Tubulopathy or other

fluid / electrolyte

imbalance (Gitelman,

Bartter, renal tubular

acidosis, Dent /

Lowe)

Unspecified cystic

kidney disease /

HNF1b nephropathy

(renal cysts and

diabetes symdrome)

I autosomal

recessive polycystic

kidney disease)

Genetics consult

only

Alport syndrome / thin

basement membrane

/ haematuria

Uromodulin-

associated

nephropathy /

autosomal dominant

tubulointerstitial

kidney disease

At-risk family

member - found to

be unaffected n

402

212

85

68

Familial hypertensive nephropathy

63

Mitochondrial nephropathy

50

Tuberous sclerosis

6
Other (Liddle, oral-facial-digital syndrome branchiooto-renal syndrome, familial hypocalciuric hypercalcaemia) 
> easy access to specialist advice, often via the specialist nurse, for patients, general practitioners and local physicians.

\section{Conclusions}

Our expectation - that this approach would enhance the overall patient/family experience, empower patients and offer opportunities for education for the family and medical trainees - has been met.

This is an adaptable model; comparable clinics have since been established by other renal departments, in both the UK and Australia.
Advances in genetics/genomics are beginning to be incorporated into everyday medical practice. As this area of science develops, its relevance and importance to current and future healthcare become more evident. It is likely that such combined clinics will proliferate, and keeping pace with progress in this field is important.

\section{Conflict of interest statement}

OW: director - UroScreen Ltd; consultant - Boston Scientific, EMS and Porges Coloplast. Grant - Porges Coloplast.

AS: hospitality recipient - Amgen, Sanofi, Otsuka. 